\title{
Características polínicas y composición química del polen apícola colectado en Cayaltí (Lambayeque - Perú)
}

\author{
Pollinic characteristics and chemical \\ composition of bee pollen collected in \\ Cayalti (Lambayeque - Peru)
}

\begin{abstract}
The aim of this study was to determine the pollen and chemical composition of four types of bee pollen, collected in The Cafetal, Cayaltí (Lambayeque, Perú), a rural area of Perú seasonally dry forest. Bee pollen was collected directly from the hives and classified into four groups of colors: yellow, orange, cream and gray. Pollen analysis revealed the presence of pollen in species: Acacia macracantha Humboldt \& Bonpland, Encelia canescens Lamarck, Momordica charantia L. and Prosopis pallida (Humboldt \& Bonpland ex Willdenow) H.B.K. The pollen of P. pallida was predominant in the gray $(98,1 \%)$ and yellow $(87,7 \%)$ bee pollen; while pollen of $E$. canecens was predominant in the orange $(72,7 \%)$ and cream (50,0\%) bee pollen, respectively. A great diversity in the pollen morphology (polyad, oblate spheroidal and prolate spheroidal), scultural elements (psilate, echinate and reticulate) and openings (tricolporate and stephanocolpate) was observed. The moisture $(8,8-13,8 \%)$, ash content $(2,1-3,2 \%)$, calcium $(6,4-12,4 \%)$, vitamin $C(208-504 \mathrm{mg})$, total and reducing sugars $(35-49,7$ y $22,4-26 \%$, respectively), fat $(0,15-0,18 \%)$ and proteins $(13,7-17,3 \%)$ was determined, showing significant variations depending on the color of bee pollen. The nutritive value was higher in the gray bee pollen reaching the value 3.51; in this color was predominant P. pallida pollen.

Key words: Chemical analysis of pollen; pollen nutritive value; pollinic characteristics; seasonally dry forest.
\end{abstract}

\section{INTRODUCCIÓN}

El polen es la estructura reproductiva masculina de las plantas superiores. La mayoría de las plantas de reproducción sexual dependen de la actividad polinizadora de los insectos, que transportan el polen de una planta a otra, tanto entre individuos de la misma especie o variedades de diferentes genotipos. En condiciones naturales las abejas juegan un rol importante en el mantenimiento de la biodiversidad de las plantas puesto que aseguran la reproducción y la diversidad genética (1). Esta relación simbiótica le permite al insecto obtener, en compensación, néctar y polen como alimento (2). En efecto, la relación entre las abejas y las plantas melíferas se cuenta entre las más importantes debido a que las abejas pueden colectar 10 a 1000 veces más polen que otros insectos; individualmente pueden ingresar y salir de la colmena 20 a 30 veces al día y los miembros de una misma colmena pueden
Kelina I. Saavedra C. Consuelo Rojas I. Guillermo E. Delgado $P$.

Facultad de Ciencias Biológicas, Universidad Nacional Pedro Ruiz Gallo, Ciudad Universitaria, Lambayeque - Perú.

Dirigir la correspondencia a: Profesor

Guillermo E. Delgado Paredes Facultad de Ciencias Biológicas Universidad Nacional Pedro Ruiz Gallo Ciudad Universitaria, Juan XXIII No 391 Lambayeque - Perú Teléfono: 0051-74-283610 E-mail: guidelg2001@yahoo.es

Este trabajo fue recibido el 25 de Septiembre de 2012 y aceptado para ser publicado el 25 de Enero de 2013.

visitar 20 a 30 millones de flores durante una estación (3).

En los últimos años el interés de la palinología, una disciplina botánica dedicada al estudio del polen y las esporas, se ha orientado hacia la melitopalinología, que es la palinología aplicada al estudio del polen apícola, la miel y todos los productos relacionados con esta actividad. El análisis microscópico del sedimento de la miel fue el primer método utilizado en la determinación del origen botánico y geográfico del polen (4) lo que por extensión comprende, también, al polen apícola, que es el resultado de la aglutinación del polen de las flores efectuada por las abejas pecoreadoras, mediante el néctar y sus propias sustancias salivares, que el hombre utiliza tras su recolección en los cazapolenes y subsiguiente elaboración (secado, limpieza y envasado) (5); como es conocido, el pecoreo es la conducta de las abejas obreras de recolectar polen y néctar de la flora apícola de un determinado lugar geográfico. 
El polen apícola es una fuente de proteínas, lípidos y vitaminas, resultando esencial para el crecimiento y desarrollo de las abejas más que para la producción de energía (6). En particular, el nitrógeno es crucial para el desarrollo de las larvas y la longevidad de las abejas adultas (7); es por ello la importancia de determinar las principales fuentes de polen de una región y su valor proteínico puesto que el polen es el mayor componente en la dieta de la abeja (8).

Los estudios sobre la composición química del polen son diversos. Los realizados sobre la caracterización bromatológica del polen apícola argentino, con el propósito de conocer si el polen apícola para uso alimentario conserva sus características nutricionales luego de las diferentes prácticas tecnológicas en su manipuleo, envasado y posterior almacenamiento (9) y otros estudios para determinar sus características generales y composición química, enfatizando en sus aspectos terapéuticos, microbiológicos y presencia de contaminantes (10). En cambio, los estudios sobre la composición química del polen apícola en una determinada región o especies en particular no son muy frecuentes; sin embargo, podemos citar como trabajos relevantes el realizado en 10 muestras de polen apícola de diferentes colores, colectadas en la región sur del Brasil, que permitió determinar su composición química y evaluación botánica, concluyéndose que debido a la diferencia de los taxa colectados, entre ellos Arecaceae, Asteraceae y Myrtaceae, los datos obtenidos pueden jugar un rol importante en la determinación del control de la calidad del polen apícola si las muestras fueran mayormente monoflorales (11). El estudio sobre la composición química del polen apícola fresco recolectado en el páramo de Misintá (Mérida) de Los Andes venezolanos, en el que se determinó los contenidos de humedad, cenizas, extracto etéreo, $\mathrm{pH}$ y proteínas de cuatro fracciones de color (amarillo, anaranjado, ocre y verde) de polen apícola, destacando el polen apícola amarillo como el más frecuente (12). Más recientemente, un estudio realizado en el polen de abejas seleccionado por colores, producido en Viana do Bolo (Ourense, en el noroeste de España), que permitió demostrar que el polen de abejas colectado en la colmena mostraba un contenido más alto de proteína respecto al contenido de proteína del polen colectado en la planta, aún cuando varios tipos de polen de estas especies vegetales se encontraban presentes en el polen de abejas (13).

El polen apícola es una mezcla de pellets de polen de diferentes colores recolectado por Apis mellifera en flores de diversas especies, removido por movimientos complejos entre las patas y los apéndices bucales, compactado con néctar en las corbículas de sus patas posteriores y transportado a la colmena (14). Para cosechar el polen de las colmenas es necesario obligar a las abejas pecoreadoras a que pasen por una rejilla de malla pequeña a fin de desenganchar el polen de las patas posteriores, que luego cae a un recipiente situado debajo de la trampa, conocido como cesta de polen (15).

El polen apícola se consume directamente o se procesa en extractos exhibiendo diversas propiedades farmacológicas y microbiológicas, debido a que es un suplemento nutricional por su contenido de vitaminas y su actividad hormonal, antibiótica (16) y antioxidante. Es utilizado en cosmetología, en la elaboración de cremas nutritivas, ungüentos, emulsiones, jabones, entre otros productos relacionados (17). La variación existente entre los componentes químicos del polen apícola es muy amplia dependiendo de las especies vegetales y las condiciones medioambientales donde se desarrollan (18). A esta variación habría que sumar el hecho que algunos países como Brasil han sido establecidas estrictas normas de control de calidad en la comercialización de los productos apícolas (19), en otros como Venezuela (12) y Perú, éstas normas aún no han sido elaboradas. En Argentina en el Art. 785 del Código Alimentario se encuentra una definición del polen, las condiciones de limpieza y secado, las características analíticas de composición y microbiológicas, rotulación y advertencias sobre alergias, conservación y fechas de elaboración y máximo de consumo (9).

En Perú, en especial en el bosque seco ecuatorial o bosque estacionalmente seco (BES), un bioma único en el mundo que comprende una franja costera de 100 a $150 \mathrm{~km}$ de ancho, que abarca desde los $0^{\circ} 30^{\prime}$ hasta los $5^{\circ} \mathrm{LS}$, desde la península de Santa Elena (Ecuador) hasta la cuenca media del río Chicama (Departamento de La Libertad), y en el valle del Marañón hasta los $9^{\circ}$ LS (20), los planes biológicos de conservación y reforestación se encuentran ligados a diversas actividades sociales, económicas y culturales que desarrollan las poblaciones rurales, entre ellas el fomento de la apicultura, debiendo para ello realizar un estudio de las especies vegetales reales y potencialmente utilizadas por la abejas así como de las características organolépticas y nutricionales de los productos apícolas obtenidos. El objetivo del presente estudio fue determinar las características polínicas y la composición química de cuatro tipos de polen apícola, agrupados en colores amarillo, anaranjado, crema y gris, colectados en El Cafetal, Cayaltí (Lambayeque, Perú), un área rural típica del bosque estacionalmente seco.

\section{MATERIAL Y MÉTODOS \\ Muestreo}

Las muestras se colectaron en el área rural El Cafetal, distrito de Cayaltí, departamento de Lambayeque - Perú (figura 1) entre octubre a marzo del 2000. La colecta se realizó aleatoriamente, tomándose las muestras directamente de las colmenas, habiéndose colocado en la entrada o piquera un cazapolen, aparato formado por una rejilla que tapa la entrada y un cajón inferior donde cae el polen; la rejilla presentó agujeros del tamaño preciso para que pase la abeja solamente dejando caer su carga de polen al cajón. Las muestras se colectaron cada dos días durante un lapso de 10 - 15 días y este procedimiento se repitió mensualmente durante los 6 meses que abarcó el estudio; luego se llevaron a un desecador durante 12 horas a 35 - 40 oC de temperatura hasta reducir al mínimo el porcentaje de humedad. Antes del envasado las impurezas se retiraron utilizando una pinza.

Selección y análisis polínico del polen apícola

Con auxilio de un microscopio estereoscópico, (MOTIC, serie SMZ-168) se separó el polen apícola en cuatro colores: amarillo, anaranjado, crema y gris, a razón de una muestra mensual. En la evaluación sensorial se siguió la metodología propuesta por Serra et al., 1985 (22) a través de los exámenes olfativo, gustativo, táctil y visual y en el análisis granulométrico se adoptó la clasificación de Baldi et al., 2004 (9). En el análisis para la preparación de las especies polínicas se utilizó el método de Erdtman o de acetólisis (23) y el montaje se realizó con bálsamo de Canadá. La descripción de los granos de polen se realizó utilizando la terminología propuesta en el Glossary of Pollen and Spore Terminology (24). En la determinación porcentual de los granos de polen se pesó $4 \mathrm{~g}$ de polen apícola y se diluyó en $10 \mathrm{~mL}$ de agua destilada, acetolizándose posteriormente; luego se tomó una alícuota y se montó en láminas portaobjetos para la observación al microscopio a 
400x, contándose un mínimo de 1000 granos de polen por lámina. El conteo se realizó por triplicado y por color del polen apícola. Las muestras polínicas se conservan en la Palinoteca de la Facultad de Ciencias Biológicas de la Universidad Nacional Pedro Ruiz Gallo de Lambayeque.

Los tipos de polen presentes en las muestras fueron identificados, contados y clasificados, de acuerdo a sus porcentajes, como polen dominante ( $\geq 45 \%)$, polen acompañante o secundario (16-45\%), polen aislado (3-15\%) y trazas de polen (1-3\%) (25). Cuando el tipo de polen representó $\geq 45 \%$ del número total de granos de polen, la muestra fue clasificada como monofloral (26).

\section{Análisis químico}

Las muestras del polen apícola fueron molidas en un mortero de porcelana antes de realizar los análisis químicos, que fueron realizados por triplicado, siguiendo varias metodologías $(9,11,27,28)$. En la determinación de la humedad se utilizó el método gravimétrico de la estufa mediante crisoles en temperatura de 105 oC durante $12 \mathrm{~h}$, expresándose los resultados en porcentaje de humedad; en la determinación de grasa se utilizó el método de Goldfisch pesando una muestra de 1,5 a 2 g conteniendo éter dietílico, expresándose los resultados en porcentaje de grasa en base seca; para los azúcares totales y reductores el método del reactivo de Ross tomando $1 \mathrm{~g}$ de la muestra y mezclándola con agua destilada y sulfito de sodio, expresándose los resultados en porcentaje de muestra en base seca; para nitrógeno y proteínas totales el método de micro Kjeldahal pesando 0,3 g de muestra, realizando la digestión con ácido sulfúrico, la titulación con ácido clorhídrico y el cálculo de proteína cruda con el factor 6,25, expresándose los resultados en porcentaje de proteínas en base seca y de cenizas el método de incineración directa en horno a 600 oC de temperatura durante $12 \mathrm{~h}$. Los resultados se expresaron en porcentaje de cenizas en base seca. En la determinación de micronutrientes como el calcio se utilizó el método complexométrico pesando 0,1 g de ceniza y agregándole unas gotas de ácido clorhídrico, expresándose los resultados en porcentaje en base seca y en la determinación de la vitamina $C$ el método de titulación visual con 2 a 6 gotas de diclorofenolindofenol, expresándose los resultados en $\mathrm{mg} / \mathrm{g}$.

El valor nutritivo de un producto alimenticio, definido como la capacidad de aportar principios alimenticios, se calculó teniendo en cuenta la composición de proteínas, grasas y carbohidratos, con base al factor isodinámico de Wilbur Olin Atwater (29).

Análisis estadístico

El análisis estadístico se realizó con el procedimiento ANOVA del paquete estadístico SAS (30), con los valores

\section{FIGURA 1}

Ubicación geográfica de la localidad de El Cafetal, Cayaltí (Lambayeque, Perú).

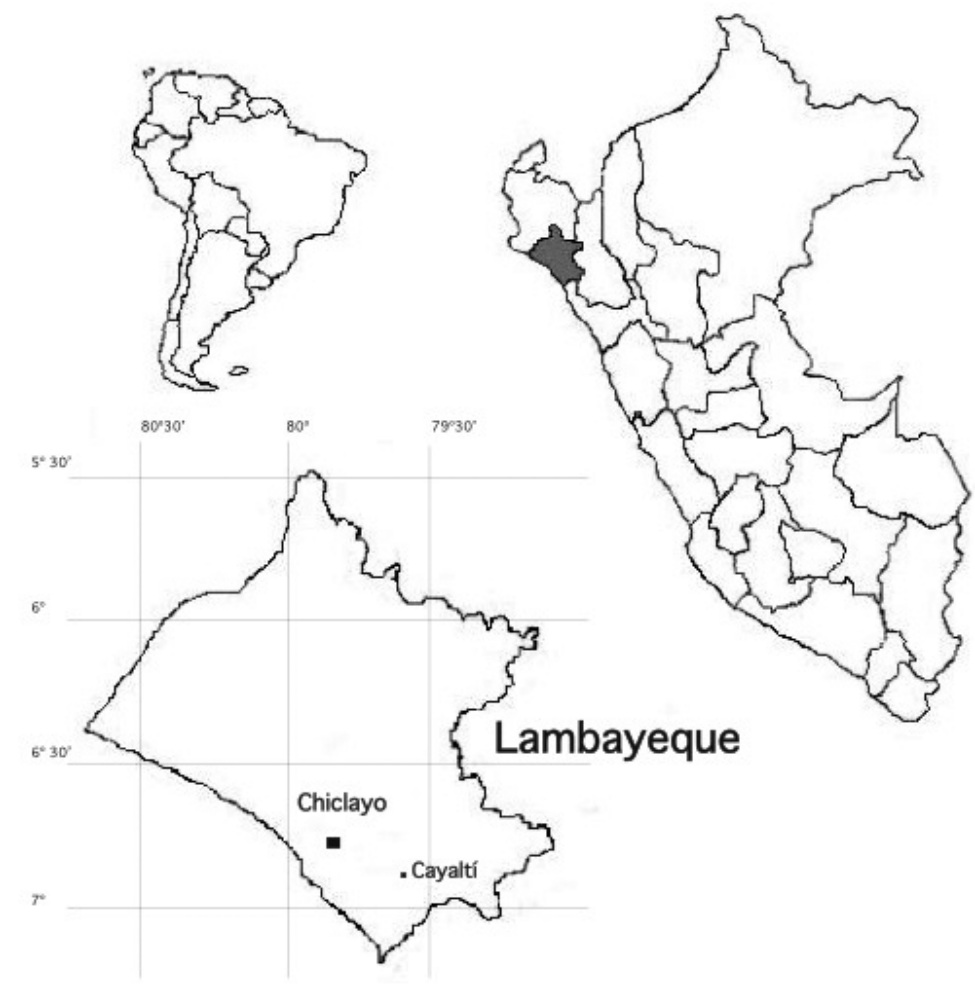


promedio por tratamiento. Para la comparación de medias se utilizó la prueba de Tukey $(P \leq 0,05)$.

\section{RESULTADOS}

Características organolécticas y granulométricas

En relación a la evaluación sensorial del polen apícola, el examen olfativo determinó un olor agradable y persistente, como atributo principal, y aromático floral, como atributo secundario; el examen gustativo determinó un sabor azucarado intenso y ácido poco intenso; el examen táctil, cuando se apretaba entre los dedos, estableció que no cambiaba de forma y cuando era mordido, que cedía sin fragmentarse (Csf), por lo tanto, podía considerarse maleable; el examen visual determinó considerarlo limpio, en tanto que el análisis granulométrico estableció tres tamaños: granos grandes $50 \%$ $(4-2,83 \mathrm{~mm})$, granos medianos $35 \%(2,83-2 \mathrm{~mm})$ y granos pequeños $15 \%$ ( $2-1,4 \mathrm{~mm})$.

\section{Determinación del polen apícola por grupo de colores}

En la tabla 1 se indica que el polen apícola, colectado a lo largo de seis meses, entre octubre a marzo del 2008, en El Cafetal, Cayaltí (Lambayeque - Perú), se clasificó en grupos de cuatro colores: amarillo, anaranjado, crema y gris. Las especies vegetales identificadas en el espectro polínico fueron: Acacia macracantha Humboldt \& Bonpland ("faique"), Encelia canescens Lamarck ("mataloba"), Momordica charantia L. ("papayilla") y Prosopis pallida (Humboldt \& Bonpland ex Willdenow) H.B.K. ("algarrobo"). En los colores amarillo y gris predominó la especie P. pallida con 87,7 y $98,1 \%$, respectivamente, en tanto que en el anaranjado y crema predominó la especie E. canescens con 72,7 y 50,0\%, respectivamente. Solamente en los colores crema y anaranjado se presentaron las cuatro especies vegetales determinadas, en tanto que en el gris y amarillo se presentaron tres y dos especies, respecti- vamente. En la mayoría de los grupos de colores, las especies A. macracantha y $M$. charantia se ubicaron en la categoría de polen aislado y ocasionalmente de polen acompañante

\section{Características polínicas de las especies vegetales}

Acacia macracantha Humboldt \& Bonpland ex Willdenow. Granos de polen en poliadas hasta 16 granos; 30,6 - 32,4 $\mu \mathrm{m}$ de diámetro; liso. La poliada está conformada por más de un grano de polen.

Encelia canescens Lamarck. Granos de polen monado; isopolar; radiosimétrico; eje polar 28,0 - $31 \mu \mathrm{m}$ y eje ecuatorial 32,4-34,2 $\mu \mathrm{m}$; P/E: 0,9 oblado esferoidal; tricolporado; espinulado.

Momordica charantia L. Grano de polen monado; isopolar; radiosimétrico; eje polar 37,3 - 39,6 $\mu \mathrm{m}$ y eje ecuatorial 34,2 - 36,4 $\mu \mathrm{m}$; P/E: 1,1 prolado esferoidal; estefanocolpado; reticulado.

Prosopis pallida (Humboldt \& Bonpland ex Willdenow) H.B.K. Grano de polen monado; isopolar; radiosimétrico; eje polar 30,2 - 32,7 $\mu \mathrm{m}$ y eje ecuatorial $20,0-23,4 \mu \mathrm{m}$; P/E: 1,4 prolado esferoidal; tricolporado; liso.

\section{Análisis químico}

En la tabla 2 se presentan los resultados promedios de tres repeticiones por grupos de colores del polen apícola para los contenidos de humedad, cenizas, calcio y vitamina C. El contenido de humedad varió entre $8,8 \%$ para el amarillo y $13,8 \%$ para el anaranjado, en tanto mostraron valores intermedios y muy similares como 11,8 y $11,9 \%$, el gris y crema, respectivamente. El contenido de cenizas varió entre 2,1\% para el gris y 3,2\% para el crema, observándose valores intermedios de 2,5 y 3,0\% para el anaranjado y amarillo, respectivamente. El contenido de calcio alcanzó valores muy variables, como $6,4 \%$ para el crema y $12,4 \%$ para el anaranjado, en tanto que valores intermedios y muy similares como 9,9 y $10,0 \%$ corres-

\section{TABLA 1}

Determinación de las especies por color y espectro polínico del polen apícola en El Cafetal, Cayaltí (Lambayeque - Perú).

Color del polen apícola

Amarillo
Anaranjado

Especie (Familia)

Prosopis pallida

(Fabaceae-Mimosoideae) Encelia canescens

(Asteraceae)

E. canescens

Momordica charantia

(Cucurbitaceae)

Acacia macracantha

(Fabaceae-Mimosoideae)

Crema

E. canescens

P. pallida

A. Macracantha

M. charantia

P. pallida

A. macracantha

E. canescens
Nombre común

Espectro polínico ${ }^{1}$ \%)

Algarrobo

Mataloba

Mataloba

72,7

Papayilla

18,2

Faique

9,1

Mataloba

50,0

Algarrobo

30,0

Faique

15,9

Papayilla

4,1

Algarrobo

98,1

Faique

1,2

Mataloba

0,7

${ }^{1}$ Polen dominante $(\geq 45 \%)$, polen acompañante o secundario (16-45\%), polen aislado (3-15\%) 
pondieron al gris y amarillo, respectivamente. Asimismo, el contenido de vitamina $C$ alcanzó valores tan extremos como 208,0 mg para el amarillo y 504,0 mg para el gris, así como valores intermedios muy diferentes como 310,0 y $400,0 \mathrm{mg}$ para el anaranjado y crema, respectivamente.

En la tabla 3 se presentan los resultados promedios de tres repeticiones por grupos de colores del polen apícola para los contenidos de azúcares totales y reductores, grasas (extracto etéreo) y proteínas, así como la determinación del valor nutritivo. El contenido de azúcares totales mostró valores extremos como $35,0 \%$ para el amarillo y $49,7 \%$ para el gris, en tanto que valores intermedios y muy similares como 43,5 y $44,8 \%$ fueron observados para el anaranjado y crema, respectivamente. Una tendencia similar se observó con los azúcares reductores donde el menor valor con 22,4\% correspondió al amarillo y el mayor valor con $26,0 \%$ al gris, en tanto que valores intermedios muy próximos a los extremos y similares entre ellos como 23,6 y $24,2 \%$ correspondieron al crema y anaranjado, respectivamente. El contenido de grasa mostró valores muy similares entre los grupos de colores donde el menor valor con $0,15 \%$ correspondió al crema y el mayor valor con $0,18 \%$ al anaranjado. Asimismo, el contenido de proteínas mostró valores muy próximos entre los diferentes grupos de colores analizados con un mínimo de 13,7\% para el amarillo y un máximo de $17,3 \%$ para el crema.

Estos contenidos de valores determinaron que el valor nutritivo del polen apícola mostrara diferencias sustanciales alcanzando 2,94 y 2,95 en el polen apícola amarillo y crema, respectivamente, y 3,46 y 3,51 en el polen apícola anaranjado y gris, respectivamente. En general, el valor nutritivo del polen apícola fue mayor en el polen apícola gris, donde predominó la especie P. pallida (algarrobo), una fabaceae emblemática en el bosque estacionalmente seco.

\section{DISCUSIÓN}

Referente al color del polen apícola, el amarillo se debería al alto porcentaje de polen de P. pallida, pero en el caso del gris, no obstante el alto porcentaje de polen de P. pallida, el amarillo resultó fuertemente atenuado por la presencia de polen de A. macracantha y E. canescens. Los colores anaranjado y crema estarían en concordancia con la presencia significativa de polen de E. canescens tal como se ha reportado de que varias especies de la familia Asteraceae tienen polen apícola de color anaranjado (31). El color del polen está determinado

\section{TABLA 2}

Contenido de humedad, cenizas, calcio y vitamina C del polen apícola en El Cafetal, Cayaltí (Lambayeque - Perú) ${ }^{1}$.

\begin{tabular}{|c|c|c|c|c|}
\hline Color del polen apícola & Humedad (\%) & Cenizas (\%) & Calcio (\%) & Vitamina C (mg) \\
\hline Amarillo & $\begin{array}{c}8,8^{c} \\
( \pm 0,54)\end{array}$ & $\begin{array}{c}3,0^{\mathrm{b}} \\
( \pm 0,06)\end{array}$ & $\begin{array}{c}10,0^{\mathrm{a}} \\
( \pm 5,7)\end{array}$ & $\begin{array}{c}208,0^{d} \\
( \pm 0,05)\end{array}$ \\
\hline Anaranjado & $\begin{array}{c}13,8^{a} \\
( \pm 2,36)\end{array}$ & $\begin{array}{c}2,5^{c} \\
( \pm 0,03)\end{array}$ & $\begin{array}{c}12,4^{b} \\
( \pm 5,7)\end{array}$ & $\begin{array}{l}310,0^{c} \\
( \pm 0,1)\end{array}$ \\
\hline Crema & $\begin{array}{c}11,9^{b} \\
( \pm 0,28)\end{array}$ & $\begin{array}{c}3,2^{\mathrm{a}} \\
( \pm 0,04)\end{array}$ & $\begin{array}{c}6,4^{c} \\
( \pm 5,7)\end{array}$ & $\begin{array}{c}400,0^{b} \\
( \pm 0,11)\end{array}$ \\
\hline Gris & $\begin{array}{c}11,8^{b} \\
( \pm 0,58)\end{array}$ & $\begin{array}{c}2,1^{d} \\
( \pm 0,04)\end{array}$ & $\begin{array}{c}9,9^{a} \\
( \pm 1,4)\end{array}$ & $\begin{array}{c}504,0^{a} \\
( \pm 0,03)\end{array}$ \\
\hline
\end{tabular}

${ }^{1}$ Los valores indican la media de tres repeticiones $\pm(S D)$. Las letras indican diferencias significativas $(p<0,05)$.

\section{TABLA 3}

Contenido de azúcares (totales y reductores), grasa (extracto etéreo), proteínas y valor nutritivo del polen apícola en El Cafetal, Cayaltí (Lambayeque - Perú) ${ }^{1}$.

\begin{tabular}{|c|c|c|c|c|c|}
\hline \multirow[t]{2}{*}{ Color } & \multicolumn{2}{|c|}{ Azúcares (\%) } & \multirow[t]{2}{*}{ Grasa (\%) } & \multirow[t]{2}{*}{ Proteínas (\%) } & \multirow[t]{2}{*}{ Valor nutritivo } \\
\hline & Totales & Reductores & & & \\
\hline Amarillo & $\begin{array}{c}35,0^{\mathrm{b}} \\
( \pm 1,45)\end{array}$ & $\begin{array}{c}22,4^{a} \\
( \pm 3,28)\end{array}$ & $\begin{array}{c}0,16^{\mathrm{b}} \\
( \pm 0,007)\end{array}$ & $\begin{array}{c}13,7^{b} \\
( \pm 0,47)\end{array}$ & $\begin{array}{c}2,94^{b} \\
( \pm 0,15)\end{array}$ \\
\hline Anaranjado & $\begin{array}{c}43,5^{a} \\
( \pm 2,24)\end{array}$ & $\begin{array}{c}24,2^{a} \\
( \pm 3,19)\end{array}$ & $\begin{array}{c}0,18^{a} \\
( \pm 0,014)\end{array}$ & $\begin{array}{c}14,3^{b} \\
( \pm 1,73)\end{array}$ & $\begin{array}{c}3,46^{a} \\
( \pm 0,28)\end{array}$ \\
\hline Crema & $\begin{array}{c}44,8^{a} \\
( \pm 0,01)\end{array}$ & $\begin{array}{c}23,6^{a} \\
( \pm 1,73)\end{array}$ & $\begin{array}{c}0,15^{\mathrm{b}} \\
( \pm 0,006)\end{array}$ & $\begin{array}{c}17,3^{a} \\
( \pm 0,76)\end{array}$ & $\begin{array}{c}2,95^{\mathrm{b}} \\
( \pm 0,24)\end{array}$ \\
\hline Gris & $\begin{array}{c}49,7^{a} \\
( \pm 1,37)\end{array}$ & $\begin{array}{c}26,0^{a} \\
( \pm 2,90)\end{array}$ & $\begin{array}{c}0,17^{a} \\
( \pm 0,004)\end{array}$ & $\begin{array}{c}16,0^{a} \\
( \pm 2,47)\end{array}$ & $\begin{array}{c}3,51^{a} \\
( \pm 0,37)\end{array}$ \\
\hline
\end{tabular}

${ }^{1}$ Los valores indican la media de tres repeticiones $\pm(S D)$. Las letras indican diferencias significativas $(p<0,05)$. 
por la presencia de pigmentos tales como flavonoides $\mathrm{y} / \mathrm{o}$ carotenoides; los flavonoides determinan los colores rojo, rosado, púrpura y azul y los carotenoides los colores amarillo pálido hasta el rojo oscuro con matices de rojo-anaranjado y amarillo-anaranjado (32).

Por lo general las abejas realizan cargas de polen monoflorales y cada carga se forma con una sola especie floral, de ahí su color uniforme; sin embargo, está influenciado por varios factores extrínsecos que determinan las variaciones de las tonalidades dentro de una misma especie $(33,34)$. En un estudio sobre polen apícola realizado en el sur del Brasil se concluyó que el color no fue determinante para la identificación de los taxa debido a que la mayoría de las muestras consideradas monoflorales presentaban dos o más taxa; entendiéndose como taxa, plural de taxón, a un grupo de individuos con posibles afinidades filogenéticas; sin embargo, se asumió, por los análisis realizados, que pueden jugar un rol importante en la determinación del control de la calidad del polen apícola si las muestras fueran mayoritariamente monoflorales (11). En el estudio que se presenta, se asume que la presencia de polen de más de una especie vegetal, en cada color estudiado, se deba a que el polen apícola del mismo color, pero con matices diferentes, correspondió a especies vegetales diferentes y su presencia en cantidades menores se deba a que correspondió a granos o bolas de polen clasificados como pequeños $(2-1,4$ $\mathrm{mm}$ ) pero que alcanzaron hasta $15 \%$ del total muestreado.

Un trabajo similar al que se presenta, realizado en el páramo de Misantá (Venezuela), determinó la presencia de cuatro grupos de colores de polen apícola: amarillo, anaranjado, ocre y verde, con frecuencias estacionales tan bajas como $2,85 \%$ en junio, para el anaranjado, y tan altas como 73,7\% en febrero, para el amarillo (12); sin embargo, en dicho trabajo no se precisó las especies vegetales que aportaron el polen transportado por las abejas. Es posible que la separación por colores sea siempre una actividad subjetiva, no obstante utilizarse escalas estándares consignadas en la guía de colores de polen apícola, que el polen de una misma especie puede presentar más de un color diferente y que diferentes especies tengan el mismo color de polen (35); sin embargo, la identificación de las especies vegetales resultará siempre un complemento imprescindible.

No se conocen trabajos sobre análisis polínicos en el polen apícola producido en el BES; no obstante, se conocen algunos trabajos realizados en ambientes ecológicos algo similares como es el caso del bosque seco del Chaco argentino donde se estableció una estrecha relación evolutiva entre la abeja Geotrigona argentina y determinadas especies de la flora circundante como Prosopis, Castela, Maytenus, Capparis, Ziziphus y Pisonia, que resultaron ser fuentes importantes tanto de polen como de néctar (36). En el BES, si bien es común la presencia de Prosopis pallida, Maytenus octogona y Capparis scabrida, únicamente el polen de P. pallida fue pecoreado por las abejas, a pesar que las otras dos especies presentaban floración en la época que se realizó el estudio; es posible que los hábitos pecoreadores entre A. mellifera y G. argentina sean significativamente diferentes. Por el contrario, el análisis polínico realizado en muestras de miel colectadas en la vegetación de Caatinga en el estado brasileño de Bahía demostró que la especie A. mellifera pecoreaba un amplio espectro de especies vegetales, aunque las mejores representadas correspondieron a las fabaceas en general y a los géneros Mimosa, Chamaecrista y Piptadenia en particular (37).

Referente al análisis químico del polen apícola, sobre el contenido de humedad $(8,8-13,8 \%)$, los resultados que se presentan concuerdan con reportes de $3,2-9,7 \%$ (9), 7,4\%
(11), 7 - 16\% (38) y 13,2 - 17,9\% (12), pero discrepan con valores tan altos como $18,8 \%$ (39) y $19-25 \%$ (40). Como ya se indicó, el polen seco debe mantener su forma y presentar una textura dura, de grano de cereal, fragmentándose sin ceder y el porcentaje de humedad influye en este estado (9). El secado excesivo y por consiguiente bajas tasas de humedad influye sobre el color natural del polen favoreciendo los procesos de pardeamiento químico, además, que es un aspecto positivo que dificulta la contaminación microbiana posterior, en tanto que altos niveles de humedad significan un riesgo importante para la conservación (41). Es posible que el menor contenido de humedad en el polen apícola estudiado en Cayaltí (Lambayeque) se deba al entorno ecológico donde se encontraban las colmenas de abejas, clasificado como bosque seco ecuatorial o BES y caracterizado porque la temperatura es muy alta (23-24 oC media anual) y las precipitaciones pluviales muy escasas (100 mm/año), aún cuando en los meses de diciembre a marzo las Iluvias suelen incrementarse (20).

Sobre el contenido de cenizas $(2,1-3,2 \%)$, los resultados concuerdan con reportes de 1,6 - 2,14\% (12) y 2,2\% (11) pero discrepan significativamente con valores altos como $4-5,1 \%$ $(18,40)$, así como con rangos tan amplios como 0,96-6,7\% (9) y $2,5-6,5 \%$ (42). En cuanto al contenido de calcio $(6,4$ - 10\%), los resultados concuerdan ligeramente con reportes de 1 -15\% (18). Asimismo, en lo referente al contenido de vitamina C (208 - $504 \mathrm{mg})$, resultados similares que van desde 152 a 640 mg (18) y 70 - 560 mg (43) también han sido reportados; asimismo, se han reportado valores de 0,2 - 13,8 $\mathrm{mg} / 100 \mathrm{~g}$ (9) y 1 - $5 \mathrm{mg} / 100 \mathrm{~g}$ (44); sin embargo, en algunos casos, como fue reportado para el polen apícola colectado en el sur de Brasil, no se determinó la presencia de vitamina C y beta-caroteno pero sí de carotenoides totales (11).

En varios trabajos se han establecido valores de $13-55$ g/100 g de carbohidratos (10), 24 - 48\% (38) y alrededor de 50\% (40), de azúcares totales, y entre 8 - 40\% (18), de azúcares reductores, que de alguna manera resultan similares con lo reportado en el trabajo que se presenta que fueron 35 $-49,7 \%$ y $22,4-26 \%$, respectivamente. En el caso del polen apícola argentino el amplio rango de variación de los niveles de glucosa $(2,44-14,3 \%)$, fructosa $(7,04-19,47 \%)$ y sacarosa $(0,43-13,33 \%)$ fue atribuido a la diversidad de las especies vegetales, la forma de recolección del polen y las condiciones de almacenamiento (9).

En lo referente al contenido de grasa $(0,15-0,18 \%)$, del trabajo que se presenta, éstos valores han resultado muy bajos si los comparamos con lo reportado para el polen apícola amarillo $(5,37 \%)$ y anaranjado $(3,13 \%)$, colectados en el páramo de Misintá (12), valores muy próximos a 1,76-6,76\% (9), 6\% (11), 5,5\% (39) y 3,5 - 6,5\% (22); asimismo, con los valores en rangos muy amplios como $0,9-14 \%$ (38) y $1-20 \%$ $(40,42)$. Se ha indicado que el polen con cantidades elevadas de grasas son buscadas selectivamente por las abejas como fuente nutritiva (45). Es posible que la concentración muy baja de grasa en el polen apícola, en el trabajo que se presenta, obedezca a factores ambientales, en especial la temperatura, la cual es superior a 22 oC en el BES; por el contrario, en lugares de clima frío las plantas almacenan almidones en elevada concentración (40).

En cuanto al contenido de proteínas (13,7 - 17,3\%), del trabajo que se presenta, también, resultó muy bajo si lo comparamos con lo reportado para el polen apícola anaranjado $(52,6 \%)$ y amarillo $(37,3 \%)$, colectados en el páramo de Misintá (12); sin embargo, estos valores devienen en inusualmente altos puesto que otros autores han reportado concentraciones 
más moderadas como $7-26 \%(38), 18-28 \%(40), 25 \%(18)$, $17,26-30,86(9), 12,6-18,8 \%(22), 31,4 \%(39), 10-40$ $\mathrm{g} / 100 \mathrm{~g}$ de materia seca (28) y $20 \%$ (11), los que se encuentran en concordancia con los resultados expresados en el trabajo que se presenta, en especial con los resultados de (22), para el polen apícola colectado en España. Un estudio reciente realizado en el sur del Caldén (Argentina) mostró que el contenido de proteína cruda de la mayoría del polen colectado por las abejas fue dramáticamente variable, con valores tan altos para el polen de Condalia microphylla y Brassicaceae con 31,9 y $30,5 \%$, respectivamente, y tan bajos como el polen de Plantago sp. y Erodium cicutarium con 13,6 y 14,2\%, respectivamente (8); resultados similares fueron observados en el polen colectado por las abejas en la localidad de Viana do Ouro (Ourense), en el noroeste de España (13).

El valor nutritivo ideal, de acuerdo a la proporción de Atwater sería de 3,8, por lo tanto, cuando es menor el alimento es considerado rico en proteínas, tal como ha sido el caso con el polen apícola analizado en nuestro trabajo.

El año 2009, los índices de pobreza total y pobreza extrema, que corresponden a la costa rural del Perú, ocupada mayoritariamente por el bosque estacionalmente seco, se encontraban en 40,6 y 9,2\%, respectivamente, y el año 2010, el índice de desnutrición para niños menores de 5 años, en $18,4 \%$ (21). Esta es la razón que determina la búsqueda de nuevas fuentes de alimentación con niveles significativos de valor nutritivo, en donde la actividad apícola, en especial la utilización del polen apícola como fuente de consumo doméstico y sus excedentes para procurarse ingresos económicos, se convierta en una nueva alternativa alimentaria y económica que se articule con la protección del bosque estacionalmente seco, un ecosistema muy frágil y permanentemente amenazado por la actividad antrópica, entendiéndose ésta como cualquier acción o intervención que el ser humano implementa sobre la faz de la tierra.

\section{RESUMEN}

El objetivo del presente estudio fue determinar las características polínicas y la composición química del polen apícola, colectado en El Cafetal, Cayaltí (Lambayeque, Perú), un área rural del bosque estacionalmente seco. El polen apícola se colectó directamente de las colmenas y se clasificó en cuatro grupos de colores: amarillo, anaranjado, crema y gris. El análisis polínico reveló la presencia de polen de las especies: Acacia macracantha Humboldt \& Bonpland, Encelia canescens Lamarck, Momordica charantia L. y Prosopis pallida (Humboldt \& Bonpland ex Willdenow) H.B.K. El polen de P. pallida predominó en el polen apícola gris $(98,1 \%)$ y amarillo $(87,7 \%)$ en tanto que el polen de E. canescens predominó en el polen apícola anaranjado $(72,7 \%)$ y crema $(50,0 \%)$. Se observó una gran diversidad en las características morfológicas del polen, en la forma (poliada, oblado esferoidal y prolado esferoidal), elementos esculturales (liso, espinado y reticulado) y aberturas (tricolporado y estefanocolpado). Se determinó los contenidos de humedad $(8,8-13,8 \%)$, cenizas $(2,1-3,2 \%)$, calcio $(6,4-12,4 \%)$, vitamina C (208 - $504 \mathrm{mg})$, azúcares totales y reductores ( $35-49,7$ y $22,4-26 \%$, respectivamente), grasas $(0,15-0,18 \%)$ y proteínas $(13,7-17,3 \%)$, observándose significativas variaciones en función del color del polen apícola. El valor nutritivo fue mayor en el polen apícola gris alcanzando el valor 3,51; en este color de polen apícola predominó P. pallida.

Palabras clave: Análisis químico del polen, bosque estacionalmente seco, características polínicas, valor nutritivo del polen.

\section{BIBLIOGRAFÍA}

1. Payette A. Les Apoïdes du Québec: Abeilles et agriculture. L'Abeille 1996; 16: 14-5.

2. Gentry C. Apiculture de petite échelle. Washington DC: Peace Corps, Info Collect \& Exch Man; Sér. 34. 1982.

3. Dongock DN, Tchoumboue J, Ricciardelli G, Youmbi E, Pinta YJ. Spectrum of melliferous plants used by Apis mellifera adansonii in the Sudano-Guinean western highlands of Cameroon. Grana 2007; 46: 123-8.

4. Silici S, Gökceoglu M. Pollen analysis of honeys from Mediterranean region of Anatolia. Grana 2007; 46: 57-64.

5. Baldi B. Influencia del proceso de secado del polen para uso alimenticio. Ciencia Docencia Tecnol 1999; 18: 241-74.

6. Schmidt JO, Buchman SL. Pollen digestion and nitrogen utilization by Apis mellifera L. (Hymenoptera: Apidae). Comp Biochem Physiol 1985; 82: 499 -503.

7. Schmidt JO, Thoenes SC, Levin MD. 1987. Survival of honey bees, Apis mellifera (Hymenoptera: Apidae), fed various pollen sources. - Ann Entomo Soc Am 1987; 80: 176-183.

8. Andrada AC, Tellería MC. Pollen collected by honey bees (Apis mellifera L.) from south of Caldén district (Argentina): botanical origin and protein content. Grana 2005; 44: 115-22.

9. Baldi B, Grasso D, Chaves S, Fernández G. Características bromatológicas del polen apícola argentino. En: Ciencia, Docencia y Tecnología Año XV 2004; 29: 145-181.

10. Mărgăoan R, Mărghitaș L, Dezmirean D, Mihai CM, Bobiș $O$. Bee collected pollen - general aspects and chemical composition. Bull UASVM Animal Scie Bio 2010; 67: 254-9.

11. Almeida-Muradian LB, Pamplona LC, Coimbra S, Barth OM. Chemical composition and botanical evaluation of dried bee pollen pellets. J Food Comp Analy 2005; 18: 105-11.

12. Vit $P$, Santiago B. Composición química de polen apícola fresco recolectado en el páramo de Misintá de los andes venezolanos. Arch Latinoam Nutr 2008; 58: 411-5.

13. De Sá-Otero MP, Armesto-Baztan S, Días-Losada E. Analysis of protein content in pollen loads produced in north-west Spain. Grana 2009; 48: 290-6.

14. Roubik DW. Ecology and Natural History of Tropical Bees. Cambridge Tropical Biology Series. Victoria:Cambridge University Press. 1992.

15. Michener CD. The corbiculae of bees. Apidologie 1999; 30: 67-74.

16. Abouda Z, Zerdani I, Kalolou I, Faid M, Ahami MT. The antibacterial activity of Moroccan bee bread and beepollen (fresh and dried) against pathogeneic bacteria. Res J Microbiol 2011; 6: 376-84.

17. Serra J, Escolá JR. Nutrient composition and microbiological quality of honey bee-collected pollen in Spain. J Agric Food Chem 1997; 45: 725-32.

18. Rojas A. Manual de Apicultura. Ed. El Águila. Cajamarca, Perú. 1992.

19. SDA. Secretário de Defesa Agropecuária. Ministerio de Agricultura, Cría y Abastecimento en Brasil. Instrução Normativa No 3, de 19 de Janeiro de 2001. Anexos V-VI. Regulamento Técnico para Fixação e Qualidade de Pólen Apícola, Própolis. Brasil. 2001.

20. Brack A, Mendiola C. Ecología del Perú. PNUD, Asociación Editorial Bruño. Lima, Perú. 2004.

21. BM (Banco Internacional de Reconstrucción y Fomento/ Banco Mundial). Perú en el umbral de una nueva era. Lecciones y desafíos para consolidar el crecimiento económico 
y un desarrollo más incluyente. Notas de Política. Vol. I. C. Felipe Jaramillo y Carlos Silva-Jáuregui (Eds.). 182 p 2010.

22. Serra Bonvehí J, Gómez-Pajuelo A, Gonell-Galindo J. Test organoleptiques du pollen em pelotes. Tech Apic 1985; 117-24.

23. Erdtman $G$. The acetolysis method, a revised description. Svensk Bot Tidskr 1960; 54: 561-4.

24. Punt $W$, Hoen PP, Blackmore S, Nilsson S, Le Thomas A. Glossary of Pollen and Spore Terminology. Rev Paleobot Palynol 2007; 143(1-2): 1-81.

25. Louveaux J, Maurizio A, Vorwhol G. Methods of melissopalynology by International Commission for bee Botany or IUBS. Bee World 1970; 51: 125-38.

26. Louveaux J, Maurizio A, Vorwhol G. Methods of melissopalynology by International Commission for bee Botany or IUBS. Bee World 1978; 59: 139-57.

27. Pearson D. Técnicas de Laboratorio para el Análisis de Alimentos. Ed. Acribia, S.A. Zaragoza, España. P. 331, 1981.

28. Campos MGR, Bogdanov S, Almeida-Muradian LB, Szczesna T, Mancebo Y, Frigerio C, Ferreira T. Pollen composition standardisation of analytical methods. J Apic Res and Bee World 2008; 47: 154-61.

29. FAO. Grasas y aceites en la nutrición humana. Consulta FAO/OMS de expertos. Roma: Estudio FAO Alimentación y Nutrición. 1993.

30. SAS. SAS/STAT user's guide. Version 6. Fourth edition. Statistical Analysis System Institute. Cary, NC, USA 846 p. 1989.

31. Vit $P$, Meléndez $P$, Schwartzenberg JC. Espelettia lenia schultzii Wedd. Ficha botánica de interés apícola en Venezuela, No. 5 Frailejón. Rev Fac Farmac 2003; 45: 80-2.

32. Montenegro G, Avila G, Rougier, Timmermann B. Pollen loads: source of carotenoids originating from the mediter- ranean plant communities of the central zone of Chile. Rev Chil Hist Nat 1997; 70: 91-9.

33. Louveaux J. Recherces sur la recolte du polen par les abeilles (Apis Mellifica L.) (fin). En: Annales de L'Abeille series Cbis. Ann L'Institut Nat Recher Agron 1958; 3: 113-220.

34. Louveaux J. 1959. Recherces sur la recolte du polen par les abeilles (Apis Mellifica L.) (fin). En: Annales de L'Abeille series Cbis. Ann L'Institut Nat Recher Agron 1959; 1: 13-108.

35. Kirk W. A color guide to pollen loads of the honey bee. Cardiff: International Bee Res Assoc 1994.

36. Vossler FG, Tellería MC, Cunningham M. Floral resources foraged by Geotrigona argentina (Apidae, Meliponini) in the Argentine Dry Chaco forest. Grana 2010; 49: 142-53.

37. Pereira P, Van den Berg C, Ribeiro F. Pollen analysis of honeys from Caatinga vegetation of the state of Bahia, Brazil. Grana 2010; 49: 66-75.

38. Valla J. Morfología de las Plantas Superiores. Edit. Hemisferio Sur, Argentina. 1983.

39. Human H, Nicolson SW. Nutritional content of fresh, beecollected and stored pollen of Aloe greatheadii var. davyana (Asphodelaceae). Phytochemistry 2006; 67: 1486-92.

40. Sáenz C. Polen y Esporas. Edit. Blume, Madrid. 1978.

41. Serra J, Père A. 1986. Études microbiologiques du pollen d'adeilles. Ann Fals Exp Chim 1986; 849: 259-66.

42. Stanley R, Linskens H. Pollen: Biology, Biochemistry, Management. Springer-Verlag, Heidelberg, New York. 1974.

43. Mărghitaș LA. Albinele si produsele lor. Ed. Ceres. 2005.

44. Bravo R. Estudios bromatológicos del polen apícola. Tesis doctoral. Universidad Complutense de Madrid, Madrid, España. 1994.

45. Singh S, Saini K, Jain KL. Quantitative comparison of lipids in some pollens and their phagostimulatory effects in honeybees. J Apic Res 1999; 38: 87-92. 